\title{
Research Paper: Effects of Combined Mental and Physical Practices on Walking and Daily Living Activities in Individuals With Multiple Sclerosis
}

\author{
Mahsa Mohammadzadeh $^{1}$ (D), Hojjat Allah Haghgoo ${ }^{* *}$ (D), Akbar Biglarian² (D) \\ 1. Department of Occupational Therapy, University of Social Welfare and Rehabilitation Sciences, Tehran, Iran. \\ 2. Department of Biostatistic, University of Social Welfare and Rehabilitation Sciences, Tehran, Iran.
}

\begin{tabular}{|c|c|}
\hline $\begin{array}{l}\text { Use your device to scan } \\
\text { and read the article online }\end{array}$ & \\
\hline arista & $\begin{array}{l}\text { citation: Mohammadzadeh M, Haghgoo HA, Biglarian A. Effects of Combined Mental and Physical Practices on Walking } \\
\text { and Daily Living Activities in Individuals With Multiple Sclerosis. Iranian Rehabilitation Journal. 2020; 18(4):455-464. http:// } \\
\text { dx.doi.org/10.32598/irj.18.4.1070.1 }\end{array}$ \\
\hline 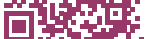 & dol'http://dx.doi.org/10.32598/irj.18.4.1070.1 \\
\hline
\end{tabular}

Article info:

Received: 03 Feb 2020

Accepted: 01 Aug 2020

Available Online: 01 Dec 2020

Keywords:

Multiple sclerosis, Walking,

Daily living activities,

Occupational therapy,

Rehabilitation, Mental practice

\section{A B S T RACT}

Objectives: Mental practice, as a neuropsychological factor effective in motor recovery, is a cognitive rehearsal of a physical skill without muscular activity. Considering the high level of fatigue in patients with Multiple Sclerosis (MS), we hypothesized that using mental practice as a low-level energy-consuming method added to physical practice could be a useful therapeutic strategy. Therefore, the current study aimed to investigate the effects of combined mental and physical practices on walking and daily living activities in patients with MS.

Methods: A randomized double-blind controlled trial was applied in the present research. In total, 22 subjects with MS were randomly allocated into the occupational therapy and mental practice groups; all study subjects received equal occupational therapy interventions 3 days a week for 6 weeks. However, in addition to occupational therapy services, the study group received mental exercises. Such practices included the visual and kinesthetic imagery of walking activity in the presence of external cues. Walking ability and daily living activities were assessed at pre-treatment, post-treatment, and 2 weeks after the treatment (follow-up). Gait parameters (distance and speed) were measured by the functional scales of the 6-Minute Walk Test and the Timed 25-Foot Walk Test. The Barthel Index was used to test individuals' performance in daily living activities.

Results: The presented combined mental and physical practice significantly improved walking distance and walking speed in post-treatment $(\mathrm{P}=0.047, \mathrm{P}<0.001)$ and follow-up $(\mathrm{P}=0.044, \mathrm{P}=0.001)$ assessments, respectively. The Barthel Index scores significantly changed per group; however, no significant differences were found between the control and test groups in this regard $(\mathrm{P}=0.386)$

Discussion: The present study data revealed that performing mental practice along with occupational therapy interventions are more effective than regular interventions alone in the gait rehabilitation of patients with MS. These significant differences in walking performance in the intervention group remained obvious till the follow-up stage. 


\section{Highlights}

- The combined mental and physical practice is more effective for improving gait function than physical practice alone.

- Incorporating mental practice into rehabilitation had no significant effect on improving the ability to performing daily living activities, compared with using rehabilitation alone.

\section{Plain Language Summary}

Multiple Sclerosis (MS) challenges a person's gait ability and Activities of Daily Living (ADL). Recently, the mental practice has been among the most widely used methods to enhance gait function in people with neurological conditions. Mental practice is a symbolic exercise involving repeated mental imagery in the absence of muscle contraction. This paper investigated the effect of mental practice in combination with physical practice on walking and ADL performances in these patients. The related result suggested that combined physical and mental practice can improve walking functioning in patients with MS, although it did not significantly change their ADL performance.

\section{Introduction}

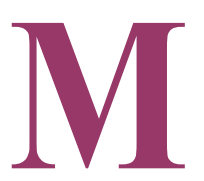

ultiple Sclerosis (MS) is a chronic and progressive autoimmune disease that affects the Central Nervous System (CNS). The major pathology of MS is the inflammation, demyelination, and degeneration of sensory-motor axons in the CNS [1]. Given the extent and severity of the affected area, the type and severity of the symptoms differ [2]. These signs and symptoms include spasticity or muscle weakness, fatigue, discoordination, ataxia, pain, sensory disturbances, gait disorders, and so on; such conditions have been repeatedly reported in these patients $[3,4]$.

Walking is a complex sensory-motor function that relies on the simultaneous interaction of the motor system, sensory control, and cognitive functions [5]. Gait disorder often results from a combination of symptoms, such as fatigue, weakness, spasticity, ataxia, and imbalance [6]. Such a condition is present in $85 \%$ of patients with MS, as their main complaints [3]. These disorders affect the level of activity, participation, and quality of life in patients with MS and result in losing the ability to work [7]; increased odds of falling and related injuries [8], as well as the patient's increased dependence on others for performing the Activities of Daily Living (ADL) [9]. The rehabilitation interventions used to overcome such debilitating gait abnormalities include resistive exercises, aerobic, traction, balance, and other exercises [1]. These interventions are often accompanied by problems, such as increased body temperature, abnormal fatigue, and exacerbated neurological symptoms. Thus, many of these patients may refrain from therapeutic exercises [10].
Applying appropriate therapeutic strategies seem necessary to manage walking problems and ADL in patients with MS $[9,11]$. Mental Practice (MP) or Motor Imagery (MI) is among the recommended strategies to enhance motor performance [12]. MP is a symbolic exercise, involving repeated mental imagery in the absence of any visible muscle contraction, i.e., performed to improve motor performance [13]. Several studies suggested that MP can be a promising additional rehabilitation method for patients with neurological disorders $[14,15]$. Performing physical practice plus mental training is supported to be more effective than merely one of the practices alone $[15,16]$. Besides, the impact of physical exercise is promoted when added to MP [17]. Several investigations have examined the incorporation of physical exercise into the MI of functional mobility in stroke survivors or patients with Parkinson's disease. For instance, Hosseini et al. reported that adding MP to current therapy could improve postural balance in stroke patients [15]. Furthermore, EI-Wishly et al. reported significant improvement in gait ability following the receipt of MI plus physical practice in patients with Parkinson's disease [14].

Most evidence in stroke patients indicated that MP may also improve ADL. Liu et al. illustrated the positive impact of employing MI training on promoting the patients' relearning of ADL. According to this report, the relearning may be attributed to the improved attention, working memory, and a higher level of processing ability of patients who underwent $\mathrm{MI}$; all are essential for conducting ADL [18]. 
Patients with MS and cognitive impairments encounter difficulties in MP. Moreover, cognitive impairments in these patients reduce the beneficial effects of mental exercises; however, MP is performed using internal (kinesthetic) and external (visual) imaging in this population. Using external cues while performing MP improves the quality of MI and facilitates MP [19, 20]. Using external cues accompanying with MP for upper extremity movements in patients with MS, researchers reported that this technique can be a valuable therapy for this group [19].

Most studies have examined the effects of MP with or without physical practice on healthy subjects and stroke patients $[15,21]$. Besides, only two studies investigated the effects of mental practice alone, using external cues on gait performance in patients with MS [20, 22]. However, no study has specifically examined the effects of combined physical and mental practice in subjects with MS. Furthermore, considering the crucial role of gait in ADL performance, no research has been conducted to examine the effects of this therapeutic approach on ADL in this population. Therefore, the present study aimed to examine the effects of combined MP and occupational therapy interventions on gait function and ADL in patients with MS.

\section{Methods}

This randomized double-blind controlled trial was conducted in Abolfazl Rehabilitation Clinic and Raha Rehabilitation Center in Isfahan City, Iran, in 2017. In total, 22 patients with MS were equally divided into the control and MP groups. The study participants were blind to the information of the two groups. The evaluations were performed in pre-treatment, post-treatment, and 2 weeks after the treatment (follow-up) in a blinded manner for the examiner. The assessments were conducted by a certified occupational therapist, blind to the control and experimental subjects.

A total of 38 patients with MS were referred to the Rehabilitation Clinic of MS and the Rehabilitation Center in Isfahan. After the initial assessment and considering the inclusion and exclusion criteria of the research, 26 patients were evaluated in detail. The inclusion criteria of the study included receiving a definite diagnosis of MS, including all 4 types of MS disease (by a neurologist); an age range of 20 to 60 years; Expanded Disability Status Scale (EDSS) rating scores ranging between 1.5 and 4.5; obtaining Mini-Mental State Examination (MMSE) scores of $>24$ [23]; a visual imaging and sensory-motor imaging score of $\geq 25$ [24]; being at remission period; the lack of deformity, and the absence of other simultaneous illnesses, such as severe visual impairment and severe orthopedic problems in the lower extremities. In the case of suffering from acute depression disorder, pregnancy, lactation, and the recurrence of disease during the intervention period, the subjects were excluded from the study. Therefore, two individuals were excluded from the study due to recurrence of the disease before initiating the study, and two were excluded at the midintervention because of orthopedic problems.

Finally, 22 subjects $(\mathrm{F}=17)$ were re-evaluated immediately after the intervention and 2 weeks after the last intervention session (follow-up). Among 22 participants, 13 had relapsing-remitting MS. Of the total 22 participants, 11 subjects received immunosuppressant drugs, such as fingolimod and natalizumab, 4 research subjects were treated with interferon beta and alpha; 4 subjects were treated with muscle relaxant drugs, such as baclofen, and 1 individual was treated with herbal medicines; however, 2 study subjects received no medication.

In the present study, 6 tools were used to collect the required information. In the first stage, the EDSS was applied to assess the severity of the disease and the degree of disability in the study participants [25]. The EDSS is a valuable outcome assessment to use in MS clinical trials with an excellent test-retest reliability $(\mathrm{r}=0.93)$ [26]. Besides, it presented a high validity through correlation with the Barthel Index ( $r=0.89)$ [27]. The MMSE is a 30item questionnaire extensively applied in clinical and research settings to measure cognitive impairment, including orientation, concentration, attention, verbal memory, naming, and visuospatial skills [23].

The 10-item Kinesthetic and Visual Imagery Questionnaire (KVIQ) was used to evaluate the research subjects' ability to imagine [24], comprising 10 questions (5 gestures for both dimensions). In the KVIQ, individuals are initially requested to perform a specific action, followed by the immediate visualization of the same action; eventually, they are requested to assess their mental imagery ability in visual and kinesthetic dimensions on two 5-point subscales [28]. This questionnaire was standardized in Iran with the test-retest reliability and an IntraClass Correlation Coefficient (ICC) of between 0.76 and 0.84 in patients with MS [29].

Furthermore, the 6-Minute Walk Test (6MWT) was applied to evaluate the distance traveled by the patient during 6 minutes [30]. Then, the Timed 25-Foot Walk Test (T25FWT) was used to measure the time to travel 25 feet [31]. According to evidence, improvements in walking speed [31] and walking distance [32] are considered clin- 
ically significant if being $\geq 20 \%$ more than the baseline rate. Finally, the Barthel Index (BI) was used to determine the patients' independence in performing ADL [33].

The study participants were randomly assigned into two groups of control and experiment through a simple random sampling method. All research subjects received occupational therapy exercises, including lower limb stretching, strengthening exercises, balance exercises, walking training for 45 (the experimental group) or 60 minutes (control group) per session. The experimental group additionally participated in MP for another 15 to 20 minutes (per session) by another certified occupational therapist. The course of treatment for all study subjects was 3 times/week for 6 weeks.

The MP interventions for gait were based on the research performed by Dunsky et al. [34]. All sessions were performed while the study subjects individually laid down on a comfortable bed or therapeutic mat in a quiet room with their eyes closed. Each session started with 5 minutes of relaxation exercises. MI training was performed for the next 10 minutes. It consisted of visual ("viewing" themselves performing the task through the eyes of a third-person perspective) and kinesthetic ("feeling" the bodily sensations of performing the task that the person can experience in the real situation) MI of the walking performance using external cues; the same was first provided by a therapist (verbal cues) and then by a metronome. During the visual and kinesthetic imagery, the research subjects were requested to visualize themselves in both dimensions while walking in the straight lane, as well as focusing on steps and walking speed in the presence of verbal cues. Precisely, they were requested to first focus on the left steps followed by the right steps, subsequently both steps. Next, they were requested to adjust their walking speed to the rhythm of a metronome. Finally, the intervention was finished by performing relaxation practices for 3-5 minutes.

The normality and homogeneity of the data collected from the walking function by $6 \mathrm{MWT}$ and T25FWT tests, as well as BI were established in the study groups. The tests were conducted in pre-test, post-test, and follow-up stages. Using the Shapiro-Wilk test and Independent Samples t-test, the mean pre-test, post-test-follow-up scores of the research groups were compared using the Independent Samples t-test. Moreover, the one-way Analysis of Covariance (ANCOVA)was used to investigate the pre-test, post-test differences of evaluated variables among the study groups. All statistical analyses were performed using SPSS at a significance level of 0.05 .

\section{Results}

A total of 22 subjects (17 females and 5 males) were eligible to participate in this research program. The Mean \pm SD age, KVIQ, and MMSE scores of the study participants were $40.32 \pm 9.12$ years, $49.45 \pm 0.96$, and $29.59 \pm 0.73$, respectively. An average KVIQ indicated excellent vividness of each dimension of motor imagery (the clarity of the image/the intensity of sensation). Furthermore, an average MMSE presented a normal cognition in both research groups. The demographic characteristics of the study participants are presented in Table 1.

According to the results of the Independent Samples t-test and Shapiro-Wilk test, the study groups were homogenous in age, disease duration, cognitive level (MMSE), disability (EDSS), the vividness of motor imagery (KVIQ-10), walking distance (6-MWT), walking speed (T25FWT), and $\mathrm{BI}(\mathrm{P}>0.05)$ before the intervention. Furthermore, the evaluated variables, such as 6-MWT, T25FWT, and BI presented a normal distribution; therefore, parametric statistics were used to analyze the collected data (Table 2).

Comparing the mean scores between the study groups suggested that in the control group, the $\mathrm{BI}(\mathrm{P}<0.01)$, 6MWT $(\mathrm{P}<0.0001)$, and T25FW $(\mathrm{P}<0.001)$ have significantly changed after the intervention. Moreover, the mean score differences of these three assessments in the control group remained statistically significant till the follow-up phase $(\mathrm{P}<0.05)$. Furthermore, in the experimental group (occupational therapy plus MP), comparing the mean scores of the three assessment stages indicated significant changes occurring after the intervention that remained until the follow-up step.

To investigate the combined effects of mental and physical practice on walking distance, walking speed, and ADL, the relevant mean scores were compared between the study groups before and after the intervention and 2 weeks after the therapy. Accordingly, no significant differences were detected between the control and MP groups before the intervention. However, MP resulted in significant positive differences between the study groups at post-intervention and follow-up assessments of 6MWT and T25FW. Comparing BI scores also revealed no significant differences caused by $\mathrm{MI}$ in the evaluation stages between the mentioned groups. These findings were supported by the one-way ANCOVA data for three functional variables. The one-way ANCOVA results for functional variables $(6 \mathrm{MWT}, \mathrm{T} 25 \mathrm{FW}$, and $\mathrm{BI})$ are presented in Table 3. 
Table 1. The group-wise demographic characteristics of the study participants

\begin{tabular}{|c|c|c|c|c|}
\hline \multirow{2}{*}{ Variable } & \multirow{2}{*}{ Sequence } & \multicolumn{2}{|c|}{ Mean $\pm S D$} & \multirow{2}{*}{$\mathbf{P}$} \\
\hline & & Experiment & Control & \\
\hline Age, $y$ & Pre-test & $39.9 \pm 9.86$ & $40.7 \pm 8.77$ & 0.839 \\
\hline Disease duration & Pre-test & $12.6 \pm 7.35$ & $11.8 \pm 6.64$ & 0.787 \\
\hline MMSE & Pre-test & $29.73 \pm 0.46$ & $29.45 \pm 1.03$ & 0.801 \\
\hline EDSS & Pre-test & $3 \pm 0.89$ & $2.5 \pm 0.82$ & 0.229 \\
\hline \multirow[t]{2}{*}{ KVIQ-10 } & Pre-test & $49.5 \pm 0.93$ & $49.3 \pm 1.02$ & 0.669 \\
\hline & Pre-test & $427.8 \pm 43.08$ & $435.9 \pm 41.19$ & 0.657 \\
\hline \multirow[t]{3}{*}{ 6MWT } & Post-test & $525.9 \pm 52.64$ & $482.2 \pm 43.57$ & 0.047 \\
\hline & Follow-up & $525.6 \pm 52.56$ & $481.4 \pm 43.40$ & 0.044 \\
\hline & Pre-test & $6.59 \pm 0.91$ & $6.32 \pm 0.97$ & 0.522 \\
\hline \multirow[t]{3}{*}{ T25FWT } & Post-test & $4.27 \pm 0.36$ & $5.33 \pm 0.70$ & 0.0001 \\
\hline & Follow-up & $4.41 \pm 0.42$ & $5.39 \pm 0.72$ & 0.001 \\
\hline & Pre-test & $92.27 \pm 6.84$ & $93.64 \pm 4.52$ & 0.733 \\
\hline \multirow[t]{2}{*}{$\mathrm{BI}$} & Post-test & $98.64 \pm 2.33$ & $97.73 \pm 2.61$ & 0.386 \\
\hline & Follow-up & $98.64 \pm 2.33$ & $97.73 \pm 2.61$ & 0.386 \\
\hline
\end{tabular}

Iranian Rehabilitation \ourna 6MWT: 6-Minute Walk Test; T25FWT: Timed 25-Foot Walk Test; BI: Barthel Index; EDSS: Expanded Disability Status Scale; KVIQ: Kinesthetic and Visual Imagery Questionnaire; MMSE: Mini-Mental State Examination; SD: Standard Deviation.

Table 2. Comparing the mean pre-test, post-test, and follow-up scores of the study groups concerning 6MWT, 25FWT, and BI

\begin{tabular}{|c|c|c|c|c|}
\hline Intervention & Phase & $\mathrm{BI}(\mathrm{P})$ & 6MWT (P) & T25FWT (P) \\
\hline \multirow{3}{*}{$\begin{array}{l}\text { Occupational Therapy } \\
\text { (OT) }\end{array}$} & Pre-test/Post-test & 0.01 & 0.0001 & 0.001 \\
\hline & Pre-test/Follow-up & 0.01 & 0.0001 & 0.001 \\
\hline & Post-test/Follow-up & 0.8 & 0.7 & 0.24 \\
\hline \multirow{3}{*}{$\begin{array}{l}\text { Occupational Therapy+ } \\
\text { Mental Practice } \\
\text { (OT+MP) }\end{array}$} & Pre-test/Post-test & 0.001 & 0.0001 & 0.0001 \\
\hline & Pre-test/Follow-up & 0.001 & 0.0001 & 0.0001 \\
\hline & Post-test/Follow-up & 0.75 & 0.85 & 0.0004 \\
\hline Sequence & - & $\mathrm{BI}(\mathrm{P})$ & 6MWT (P) & T25FWT (P) \\
\hline Pre (OT \& OT+MP) & - & 0.58746 & 0.656719 & 0.521664 \\
\hline Post (OT \& OT+MP) & - & 0.399624 & 0.046812 & 0.000259 \\
\hline Follow (OT \& OT+MP) & - & 0.399624 & 0.043774 & 0.000988 \\
\hline
\end{tabular}

6MWT: 6-Minute Walk Test; T25FWT: Timed 25-Foot Walk Test; BI: Barthel Index. 
Table 3. The one-way ANCOVA Results for $6 \mathrm{MWT}, \mathrm{T} 25 \mathrm{FWT}$, and BI in the study groups

\begin{tabular}{|c|c|c|c|c|c|c|}
\hline Parameter & Source & Sum of Squares & df & Mean of Squares & $\mathbf{F}$ & Sig. \\
\hline \multirow{5}{*}{ 6-Minute Walk test } & Intercept & 713.414 & 1 & 713.414 & 1.445 & 0.244 \\
\hline & Pre.6mwt & 37331.579 & 1 & 37331.579 & 75.626 & 0.0001 \\
\hline & Group & 14708.141 & 1 & 14708.141 & 29.796 & 0.000 \\
\hline & Error & 9379.088 & 19 & 493.636 & - & - \\
\hline & a. R Squared & \multicolumn{5}{|c|}{0.836 (Adjusted R Squared = 0.819) } \\
\hline \multirow{5}{*}{ Timed 25-Foot Walk test } & Intercept & 0.959 & 1 & 0.959 & 10.962 & 0.004 \\
\hline & Pre.t25fwt & 4.686 & 1 & 4.686 & 53.579 & 0.0001 \\
\hline & Group & 7.733 & 1 & 7.733 & 88.419 & 0.0001 \\
\hline & Error & 1.662 & 19 & 0.087 & - & - \\
\hline & a. R Squared & \multicolumn{5}{|c|}{0.868 (Adjusted R Squared $=0.854$ ) } \\
\hline \multirow{5}{*}{ Barthel Index } & Intercept & 424.854 & 1 & 424.854 & 104.558 & 0.0001 \\
\hline & Pre.BI & 45.524 & 1 & 45.524 & 11.203 & 0.003 \\
\hline & Group & 8.653 & 1 & 8.653 & 2.130 & 0.161 \\
\hline & Error & 77.204 & 19 & 4.063 & - & - \\
\hline & a. R Squared & \multicolumn{5}{|c|}{0.393 (Adjusted R Squared=0.330) } \\
\hline
\end{tabular}

Пranian Rehabilitation \ourna According to the partial Eta-Squared, $61 \%$ of changes in 6-minute walking, $82 \%$ of changes in 25 -foot walking time, and $10 \%$ of changes in Barthel Index were due to the provided intervention.

\section{Discussion}

The present study aimed to address the theory that performing MP along with occupational therapy interventions is more effective than regular interventions alone in enhancing walking and ADL performances in individuals with MS. The relevant findings declared a significant improvement in the studied patient's functional mobility triggered by the supplementary benefit of using MI.

According to the results, the presented occupational therapy intervention resulted in significant changes in walking distance (6MWT scores), walking speed (T25FW scores), and BI scores in both research groups. However, combined intervention (MP and occupational therapy) significantly altered walking in the experimental group, compared with the controls. These significant differences in walking performance in the intervention group remained obvious till the follow-up stage. Therefore, although intervention has stopped, the effect of MP remained for a long time. Furthermore, the presented interventions resulted in significant changes in the BI in both study groups; however, MP with conventional intervention had no significantly different effect from the conventional therapy. Although MP improved BI scores in the experimental group much more than that of the control group, these changes were not significant.

The positive effect of combined mental and physical practice on walking ability is not surprising; improvements achieved by this method has been demonstrated in other populations over the past decades. EI-Wishly et al. reported that combined MI training and physical therapy programs can improve gait functions in patients with Parkinson's disease. Such data indicated that the preliminary effects of MI could enhance the proficiency of subsequent physical practices [14]. Cho et al. examined the potential impacts of added MP on treadmill training in stroke survivors. They found that gait training plus MP was more effective in improving functional mobility than sole conventional interventions [35]. Hosseini et al. investigated 30 stroke patients who underwent physical practice combined with MI training. They concluded that functional mobility was increased after the interven- 
tion, and this effect was maintained after two weeks [15]. Thus, the present study outcomes were consistent with those of the aforementioned findings.

To our knowledge, no study has examined the effects of mental plus physical practice on walking and ADL in individuals with MS. The obtained findings suggested that this protocol produced significant enhancements in gait; it has the potential to be applied like other therapeutic strategies.

The achieved enhancement in walking ability can be attributed to the learning impacts of cognitive practice strategy accomplished in the particular regions of the CNS. Researchers have concluded that the simultaneous use of physical and mental practice presents the greatest impact on skills learning. Moreover, several studies illustrated that all brain areas dedicated to planning, processing, and preparation for a motion can be activated during MP, like physical exercise [19, 36-38]. However, other scholars suggested that MI relies on processes involved in planning, but not the control of movements. According to Glover [39], the brain regions responsible for motor preparation or motor planning, like the inferior parietal lobule, are more engaged during MP; however, the control regions, like the superior parietal lobule are much less involved in this respect.

Jacobson indicated that the CNS projects stimulations into the fiber muscles at the time of real execution; in MI, similar impulses are induced in the brain and muscles. Though these activities of the brain and muscles during MI are less than the activity during real motion [40]. Therefore, there is a tendency in the brain to execute imagined movements. These findings can explain the improvement in walking function in patients with MS who participated in MP.

Although the improvement in walking ability is encouraging, it was unsatisfactory to find that no significant progress occurred in ADL. First, this phenomenon is possibly related to the design of the MI protocol used in the present study. We speculate that throughout the last MP sessions, gait exercises must be incorporated into actual tasks. For example, individuals should be requested to imagine themselves walking while engaging in a variety of ADL in various conditions. Besides, this may be because the treatment duration (6 weeks) was insufficient to result in a significant difference in BI scores, compared to controls. While the dose was adequate to improve gait speed and distance, it may have required to be longer for enhancing ADL in the study participants. The small sample size was another limitation of the cur- rent study. Thus, future studies with larger sample sizes and longer examination times are suggested to test the effects of the described intervention in patients with MS.

\section{Conclusion}

The present study data suggested that the combination of MP and conventional occupational therapy effectively improved walking function in patients with MS. Therefore, MP could be used as an effective adjunct method, without fatigue to the established rehabilitation approaches for motor problems in MS. Finally, MP did not significantly change the BI scores between the study groups; however, implementing these exercises resulted in better performance of the study participants concerning ADL performances. We encourage further research to study the effect of this method on other functions and in different neurological disorders.

\section{Ethical Considerations}

\section{Compliance with ethical guidelines}

This study was approved by the Ethics Committee of the USWR.AC.IR (Code: IR.USWR.REC.1396.317). Moreover, written informed consent was signed by all research participants before the study, and the privacy rights of the human subjects were observed.

\section{Funding}

This research did not receive any specific grant from funding agencies in the public, commercial, or not-forprofit sectors.

\section{Authors' contributions}

Conceptualization: Hojjat Allah Haghgoo; Methodology: Hojjat Allah Haghgoo, Mahsa Mohammadzadeh; Investigation: Mahsa Mohammadzadeh; Data curation: Akbar Biglarian; Writing-original draft: Hojjat Allah Haghgoo, Mahsa Mohammadzadeh; Writing-review and editing: Hojjat Allah Haghgoo, Mahsa Mohammadzadeh; Supervision: Hojjat Allah Haghgoo.

\section{Conflict of interest}

The authors declared no conflicts of interest. 


\section{References}

[1] Waxman SG. Clinical course and electrophysiology of multiple sclerosis. Advances in Neurology. 1987; 47:157-84. [PMID]

[2] Haider L, Zrzavy T, Hametner S, Höftberger R, Bagnato F, Grabner G, et al. The topograpy of demyelination and neurodegeneration in the multiple sclerosis brain. Brain. 2016; 139(Pt 3):807-15. [DOI:10.1093/brain/awv398] [PMID] [PMCID]

[3] Henze T, Rieckmann P, Toyka KV. Symptomatic treatment of multiple sclerosis. Multiple Sclerosis Therapy Consensus Group (MSTCG) of the German Multiple Sclerosis Society. European Neurology. 2006; 56(2):78-105. [DOI:10.1159/000095699] [PMID]

[4] Scheinberg L, Holland N, Larocca N, Laitin P, Bennett A, Hall H. Multiple sclerosis; Earning a living. New York State Journal of Medicine. 1980; 80(9):1395-400. [PMID]

[5] Jahn K, Deutschländer A, Stephan T, Kalla R, Wiesmann M, Strupp M, et al. Imaging human supraspinal locomotor centers in brainstem and cerebellum. NeuroImage. 2008; 39(2):786-92. [DOI:10.1016/j.neuroimage.2007.09.047] [PMID]

[6] Kelleher KJ, Spence W, Solomonidis S, Apatsidis D. Ambulatory rehabilitation in multiple sclerosis. Disability and Rehabilitation. 2009; 31(20):1625-32. [DOI:10.1080/09638280902751931] [PMID]

[7] Piercy J, Rajagopalan K, Pike J, Jones EC. Burden of walking problems in MS: Analysis of caregiver and indirect costs. Neurology. 2011; 76(9): A606. [DOI: 10.1016/j.jval.2011.02.1128]

[8] Cattaneo D, De Nuzzo C, Fascia T, Macalli M, Pisoni I, Cardini R. Risks of falls in subjects with multiple sclerosis. Archives of Physical Medicine and Rehabilitation. 2002; 83(6):864-7. [DOI:10.1053/apmr.2002.32825] [PMID]

[9] Yildiz M. The impact of slower walking speed on activities of daily living in patients with multiple sclerosis. International Journal of Clinical Practice. 2012; 66(11):1088-94. [DOI:10.1111/ ijcp.12003] [PMID] [PMCID]

[10] Brown TR, Kraft GH. Exercise and rehabilitation for individuals with multiple sclerosis. Physical Medicine and Rehabilitation Clinics of North America. 2005; 16(2):513-55. [DOI:10.1016/j. pmr.2005.01.005] [PMID]

[11] LaRocca NG. Impact of walking impairment in multiple sclerosis: Perspectives of patients and care partners. The Patient. 2011； 4(3):189-201. [DOI:10.2165/11591150-000000000-00000] [PMID]

[12] Sharma N, Pomeroy VM, Baron J-C. Motor imagery a backdoor to the motor system after stroke? Stroke. 2006; 37(7):194152. [DOI:10.1161/01.STR.0000226902.43357.fc] [PMID]

[13] Weinberg RS, Gould D. Foundations of sport and exercise psychology. $6^{\text {th }}$ ed. Champaign: Human Kinetics; 2014. https:/ / books.google.com/books/about/Foundations_of_Sport_and_ Exercise_Psycho.html?id=7NqVBAAAQBAJ

[14] El-Wishy AA, Fayez ES. Effect of locomotor imagery training added to physical therapy program on gait performance in Parkinson patients: A randomized controlled study. The Egyptian Journal of Neurology, Psychiatry and Neurosurgery. 2013 50(1):31-7. https://scholar.cu.edu.eg/?q=emanfayez/publications/effect-locomotor-imagery-training-added-physical-therapy-program-gait-perform
[15] Hosseini SA, Fallahpour M, Sayadi M, Gharib M, Haghgoo H The impact of mental practice on stroke patients' postural balance. Journal of the Neurological Sciences. 2012; 322(1-2):263-7. [DOI:10.1016/j.jns.2012.07.030] [PMID]

[16] McBride ER, Rothstein AL. Mental and physical practice and the learning and retention of open and closed skills. Perceptual and Motor Skills. 1979; 49(2):359-65. [DOI:10.2466/ pms.1979.49.2.359]

[17] Janssen JJ, Sheikh AA. Enhancing athletic performance through imagery: An overview. In: Sheikh AA, Korn ER, editors, Imagery in sports and physical performance Farmingdale. United States: Baywood Publishing Company; 1994. https://www.cabdirect.org/cabdirect/abstract/20053002592

[18] Liu KP, Chan CC, Lee TM, Hui-Chan CW. Mental imagery for promoting relearning for people after stroke: A randomized controlled trial. Archives of Physical Medicine and Rehabilitation. 2004; 85(9):1403-8. [DOI:10.1016/j. apmr.2003.12.035] [PMID]

[19] Heremans E, Nieuwboer A, Spildooren J, De Bondt S, D'hooge A-M, Helsen IW, et al. Cued motor imagery in patients with multiple sclerosis. Neuroscience. 2012; 206:115-21. [DOI:10.1016/j.neuroscience.2011.12.060] [PMID]

[20] Seebacher B, Kuisma R, Glynn A, Berger T. Rhythmic cued motor imagery and walking in people with multiple sclerosis: a randomised controlled feasibility study. Pilot and Feasibility Studies. 2015; 1:25. [DOI:10.1186/s40814-015-0021-3] [PMID] [PMCID]

[21] Fontani G, Migliorini S, Benocci R, Facchini A, Casini M, Corradeschi F. Effect of mental imagery on the development of skilled motor actions. Perceptual and Motor Skills. 2007; 105(3 Pt 1):803-26. [DOI:10.2466/pms.105.3.803-826] [PMID]

[22] Seebacher B, Kuisma R, Glynn A, Berger T. The effect of rhythmic-cued motor imagery on walking, fatigue and quality of life in people with multiple sclerosis: A randomised controlled trial. Multiple Sclerosis Journal. 2017; 23(2):286-96. [DOI:10.1177/1352458516644058] [PMID]

[23] Folstein MF, Folstein SE, McHugh PR. "Mini-mental state": A practical method for grading the cognitive state of patients for the clinician. Journal of Psychiatric Research. 1975; 12(3):189-98. [DOI:10.1016/0022-3956(75)90026-6]

[24] Randhawa B, Harris S, Boyd LA. The Kinesthetic and Visual Imagery Questionnaire is a reliable tool for individuals with Parkinson disease. Journal of Neurologic Physical Therapy. 2010; 34(3):161-7. [DOI:10.1097/NPT.0b013e3181e1aa71] [PMID]

[25] Kurtzke JF. Rating neurologic impairment in multiple sclerosis an expanded disability status scale (EDSS). Neurology. 1983; 33(11):1444-52. [DOI:10.1212/WNL.33.11.1444] [PMID]

[26] Syndulko K, Ke D, Ellison GW, Baumhefner RW, Myers LW, Tourtellotte WW. Comparative evaluations of neuroperformance and clinical outcome assessments in chronic progressive multiple sclerosis: I. Reliability, validity and sensitivity to disease progression. Multiple Sclerosis Journal. 1996; 2(3):142-56. [DOI:10.1177/135245859600200305] [PMID]

[27] Hobart J, Freeman J, Thompson A. Kurtzke scales revisited: The application of psychometric methods to clinical intuition. Brain. 2000; 123(Pt 5):1027-40. [DOI:10.1093/ brain/123.5.1027] [PMID] 
[28] Malouin F, Belleville S, Richards CL, Desrosiers J, Doyon J. Working memory and mental practice outcomes after stroke. Archives of Physical Medicine and Rehabilitation. 2004; 85(2):177-83. [DOI:10.1016/S0003-9993(03)00771-8]

[29] Kharestani M, Ghotbi Varzaneh A, Esmaeeli Abdar M. [The Effect of mental imagery and physical exercise on patient's static balance with Multiple Sclerosis (Persian)]. Journal of Research in Rehabilitation Sciences. 2014; 10(7):866-74. [DOI: 10.22122/jrrs.v10i7.1858]

[30] Goldman MD, Marrie RA, Cohen JA. Evaluation of the six-minute walk in multiple sclerosis subjects and healthy controls. Multiple Sclerosis. 2007; 14(3):383-90. [DOI:10.1177/1352458507082607] [PMID]

[31] Hobart J, Blight AR, Goodman A, Lynn F, Putzki N. Timed 25-Foot Walk Direct evidence that improving 20\% or greater is clinically meaningful in MS. Neurology. 2013; 80(16):1509-17. [DOI:10.1212/WNL.0b013e31828cf7f3] [PMID]

[32] Learmonth YC, Dlugonski DD, Pilutti LA, Sandroff BM, Motl RW. The reliability, precision and clinically meaningful change of walking assessments in multiple sclerosis. Multiple Sclerosis Journal. 2013; 19(13):1784-91. [DOI:10.1177/1352458513483890] [PMID]

[33] Liu W, Unick J, Galik E, Resnick B. Barthel index of activities of daily living: Item response theory analysis of ratings for long-term care residents. Nursing Research. 2015; 64(2):88-99. [DOI:10.1097/NNR.0000000000000072] [PMID]

[34] Dunsky A, Dickstein R, Marcovitz E, Levy S, Deutsch JE. Home-based motor imagery training for gait rehabilitation of people with chronic poststroke hemiparesis. Archives of Physical Medicine and Rehabilitation. 2008; 89(8):1580-8. [DOI:10.1016/j.apmr.2007.12.039] [PMID]

[35] Cho H-y, Kim J-s, Lee G-C. Effects of motor imagery training on balance and gait abilities in post-stroke patients: A randomized controlled trial. Clinical Rehabilitation. 2013; 27(8):675-80. [DOI:10.1177/0269215512464702] [PMID]

[36] Feltz DL, Landers DM. The effects of mental practice on motor skill learning and performance: A meta-analysis. In: e Smith D, Bar-Eli M, editors. Essential readings in sport and exercise psychology. Champaign: Human Kinetics; 2007. https:// psycnet.apa.org/record/2007-07146-023

[37] Gandevia SC, Wilson LR, Inglis JT, Burke D. Mental rehearsal of motor tasks recruits alpha-motoneurones but fails to recruit human fusimotor neurones selectively. The Journal of Physiology. 1997; 505( Pt 1):259-66. [DOI:10.1111/j.14697793.1997.259bc.x] [PMID] [PMCID]

[38] Papadelis C, Kourtidou-Papadeli C, Bamidis P, Albani M. Effects of imagery training on cognitive performance and use of physiological measures as an assessment tool of mental effort. Brain and Cognition. 2007; 64(1):74-85. [DOI:10.1016/j. bandc.2007.01.001] [PMID]

[39] Glover S. Separate visual representations in the planning and control of action. Behavioral and Brain Sciences. 2004; 27(1):3-24. [DOI:10.1017/S0140525X04000020] [PMID]

[40] Jacobson E. Electrophysiology of mental activities. The American Journal of Psychology. 1932; 44(4):677-94. [DOI:10.2307/1414531] 
This Page Intentionally Left Blank 